Revue des patrimoines

\title{
Travailler avec des chevaux de trait aujourd'hui : héritage, innovation, transmission
}

\section{Bernadette Lizet, Deny Fady, Ramon Garcia et Vincent Seïté}

\section{(2) OpenEdition}

\section{Journals}

Édition électronique

URL : http://journals.openedition.org/insitu/12213

DOI : $10.4000 /$ insitu. 12213

ISSN : $1630-7305$

\section{Éditeur}

Ministère de la culture

Référence électronique

Bernadette Lizet, Deny Fady, Ramon Garcia et Vincent Seïté, « Travailler avec des chevaux de trait aujourd'hui : héritage, innovation, transmission », In Situ [En ligne], 27 | 2015, mis en ligne le 29 octobre 2015, consulté le 19 avril 2019. URL : http://journals.openedition.org/insitu/12213 ; DOI : 10.4000/ insitu. 12213

Ce document a été généré automatiquement le 19 avril 2019

\section{(i) $९$}

In Situ Revues des patrimoines est mis à disposition selon les termes de la licence Creative Commons Attribution - Pas d'Utilisation Commerciale - Pas de Modification 4.0 International. 


\title{
Travailler avec des chevaux de trait aujourd'hui : héritage, innovation, transmission
}

\author{
Bernadette Lizet, Deny Fady, Ramon Garcia et Vincent Seïté
}

1 Faire le choix de travailler en France aujourd'hui en s'appuyant sur la force des animaux (des chevaux de trait, des ânes, des mulets, des bovins plus rarement) implique une motivation patrimoniale à plusieurs titres. Les hommes et les femmes qui ont recours à la traction animale partagent en effet le désir de sauvegarder les races (en France, neuf chez les chevaux de trait, sept chez les ânes), mais aussi le système constitué par le matériel (le harnachement, les outils attelés) et les compétences mobilisées dans la relation entre les hommes et leurs chevaux. Ces nouveaux professionnels sont animés par le souci de ménager la nature, prendre soin des sols, considérer l'effet de leur action sur les milieux et les espèces. Ils aiment s'immerger dans les paysages qui reflètent cette qualité de lien entre les hommes et le vivant ${ }^{1}$, une qualité de liens des hommes entre eux.

2 La fibre patrimoniale est d'autant plus forte que la motorisation agricole des Trente Glorieuses avait confiné l'activité traditionnelle dans des marges très fragiles. L'une des particularités de l'agriculture française est d'avoir longtemps conservé la petite propriété familiale, qui allait de pair avec la traction chevaline, notamment dans les cultures spécialisées, vigne et maraîchage. Mais les effectifs de chevaux n'ont cependant pas cessé de diminuer depuis l'entre-deux guerres et l'évolution s'est accélérée dans les années $1960-1970^{2}$. Une première politique nationale dite de "relance» a été alors élaborée, sur deux plans très inégaux dans les moyens mis en œuvre. L'objectif principal était de construire une filière de boucherie : le 23 juillet 1976, un arrêté ministériel rayait l'expression «cheval de trait», pour lui substituer celle de "cheval lourd». Parallèlement, un soutien était apporté à l'expérimentation néo-rurale sur la traction animale, ânes, chevaux et mulets, dans la perspective d'un transfert de compétences vers les pays en voie de développement. Une deuxième relance, très contradictoire, est intervenue dans les années 1990, avec cette fois «l'utilisation » comme mot d'ordre et 
l'incitation à intégrer le monde de l'attelage de loisir et de sport ${ }^{3}$. Toute une série d'épreuves et de compétitions subventionnées, parfois intensément médiatisées comme la Route du poisson, en ont assuré le succès ${ }^{4}$.

3 Une trentaine d'années se sont écoulées, les anciens n'ont pas lâché et une nouvelle génération de professionnels de la traction animale est aujourd'hui à l'œuvre. En témoignent trois portraits. Vincent Seïté est « débardeur », Deny Fady " utilisateur » et Ramon Garcia, «laboureur». La diversité des termes de métier signale d'emblée la diversité des modes de réinvestissement. L'objectif est de comprendre cette diversité, mais aussi de repérer les traits communs entre les trois personnages. Ce processus de réinvention durable invite à réfléchir au patrimoine sous l'angle d'une combinaison entre l'ancien et le nouveau, entre la conservation, l'expérimentation et l'innovation, entre l'activité productive et le loisir également.

\section{Débarder en Bretagne}

On a coutume de considérer la Bretagne comme une région très pauvre en forêts. Elles sont effectivement rares, et entretenues à ce titre avec beaucoup de soin par la puissance publique (l'office national des forêts) et les collectivités territoriales.

5 En 2007, après un brevet de technicien supérieur en aménagement du territoire (spécialité gestion forestière), Vincent Seïté monte à Plouigneau, dans le Finistère, une société de « Services soutien à exploitation forestière ». L'intégralité de son travail se fait avec son père, Jean-Jacques, qui s'est extirpé en 1998 d'une ferme industrielle en GAEC (Groupement agricole d'exploitation en commun), une usine à lait et à cochons, pour créer une petite entreprise individuelle de débardage avec des chevaux. Au départ, ce sont des contrats avec l'ONF qui faisaient vivre le père ; il employait un bûcheron au coup par coup sur les chantiers. À la fin des années 2000, l'affaire se développe et les partenariats se diversifient (ONF, conseils généraux, communes et communautés de communes, syndicats mixtes et un peu de privé) : Vincent peut s'installer.

6 L'écurie (père et fils) comprend aujourd'hui 25 chevaux (adultes et poulains) dont 8 de travail. Tous de race bretonne, chez laquelle on distingue bien le type «trait» qui caractérise l'écurie de travail, et le type "postier» apprécié pour l'attelage sportif, pratiqué avec passion. Quelques chevaux sont polyvalents (débardage et sport). Ils ont tous deux leur "galop $7^{5}$ ", exigé dans la compétition nationale: les Seïté sont donc débardeurs et meneurs (surtout le fils) (fig. 1, fig. 2). 
Figure 1

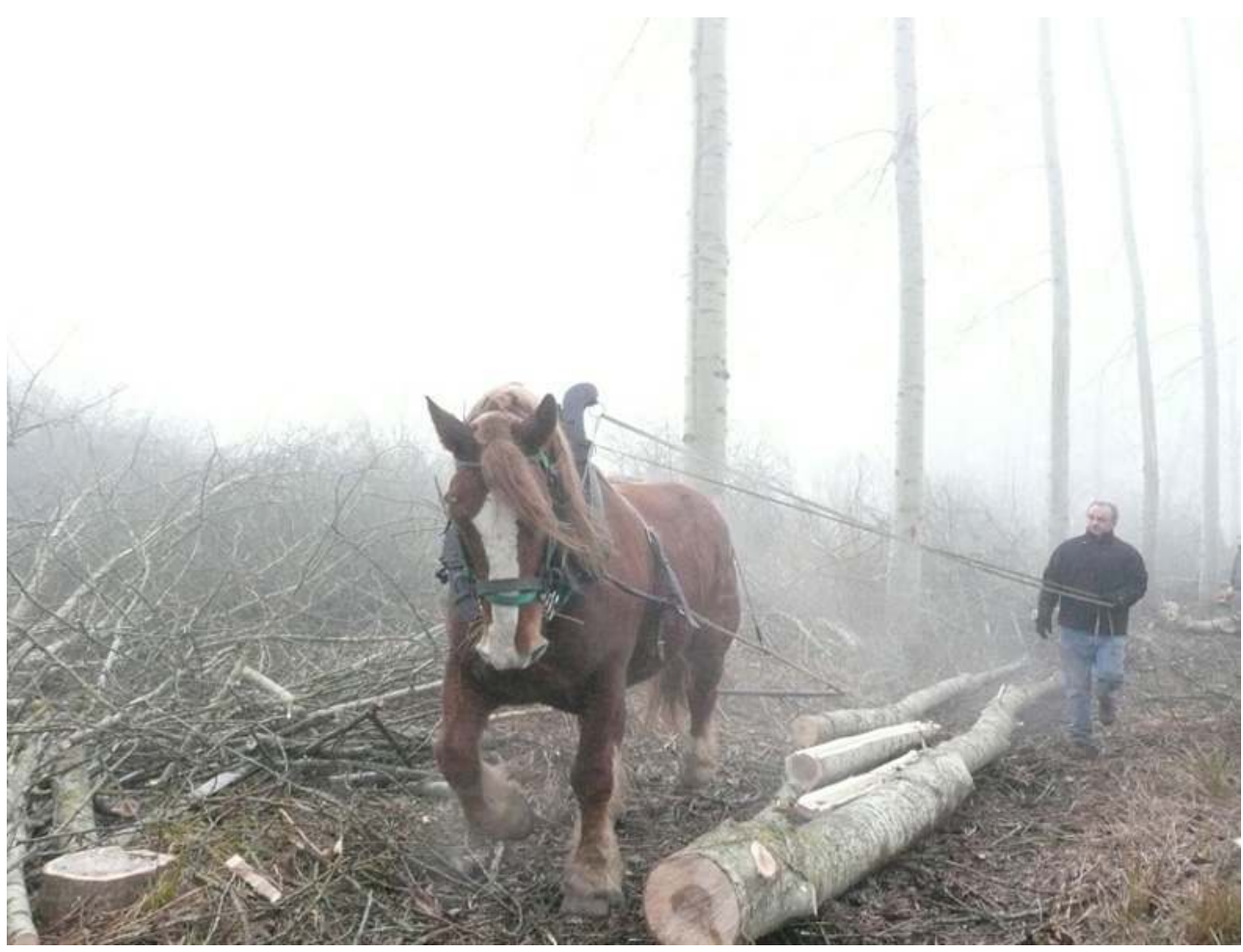

Jean-Jacques Seïté au Marais de Larchant (Seine-et-Marne) : débardage de peupliers sur une digue, le 28 janvier 2008.

Phot. Lizet, Bernadette. (c) Bernadette Lizet.

Figure 2

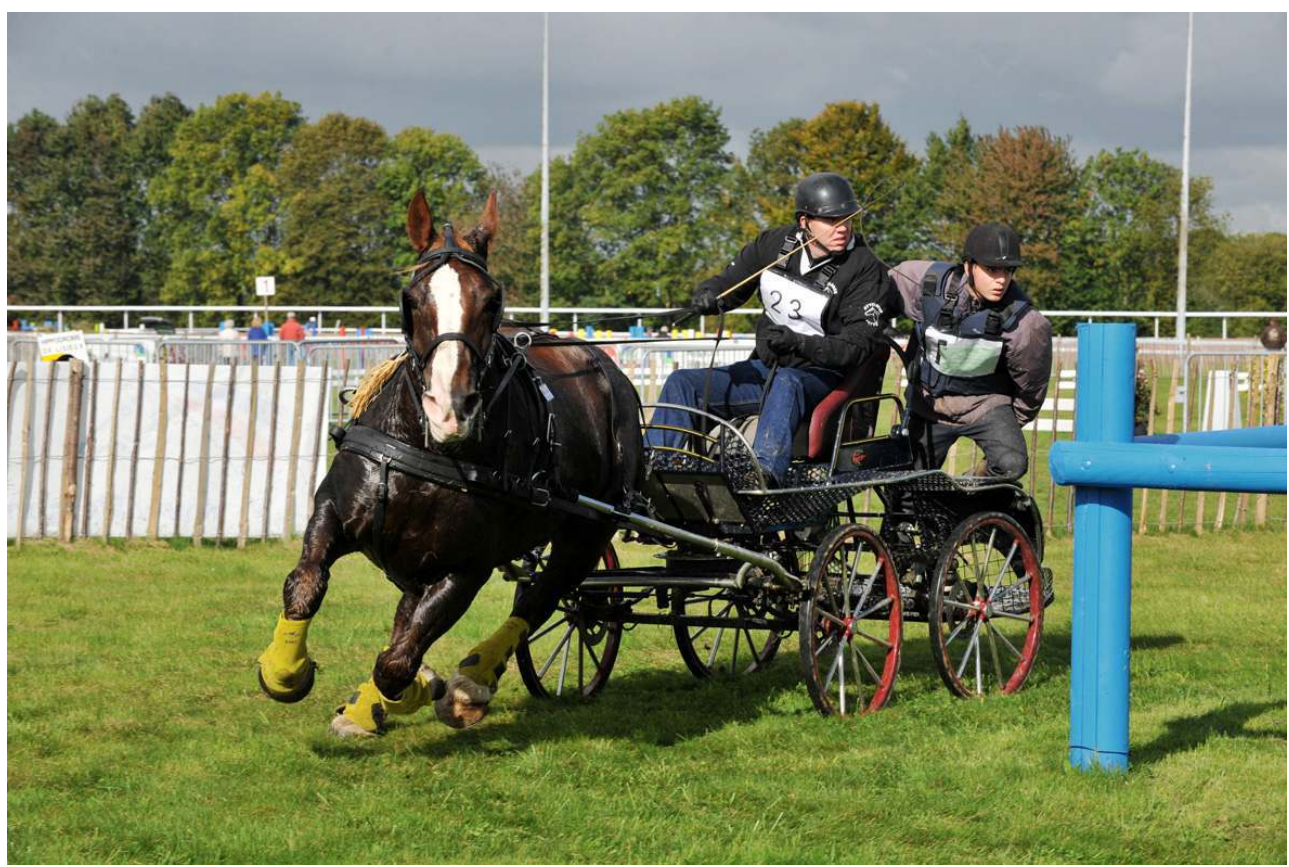

Vincent Seïté et Nevez lewanamzer dans un obstacle de l'épreuve de marathon lors du championnat de France d'attelages de chevaux de trait en 2012. (Lisieux, Calvados).

Phot. Dugast, Jean-Léo. (c) Jean-Léo Dugast. 
7 Le " cœur de métier ", c'est l'arbre et la forêt. Il y a le débardage à proprement parler : sortir du bois de taille et de valorisation différentes (bois d'œuvre et de chauffage, boisénergie: copeaux chaudière). Le recours au cheval est réservé aux secteurs difficiles d'accès et en forte pente. Mais il y a aussi et surtout le "dessouchage ", l'arrachage des souches d'arbres. Les Seïté sont devenus des spécialistes : $60 \%$ de leur chiffre d'affaire est aujourd'hui assuré par l'extraction d'une espèce exotique très conquérante, échappée des haies de pavillons : le laurier palme. L'ONF avait tenté de contrôler le laurier palme en le coupant. Avec de très mauvais résultats : l'année suivante, les arbres étaient repartis de plus belle. Deux appels d'offre ont ainsi été programmés sur quatre ans (2008-2012), financés par le conseil général du Finistère puis par celui du Morbihan, avec l'ONF comme maître d'œuvre. Les Seïté ont appris ce nouveau métier sur de jeunes sujets et aujourd'hui ils savent faire, ils s'attaquent à des arbres (fig. 3).

Figure 3

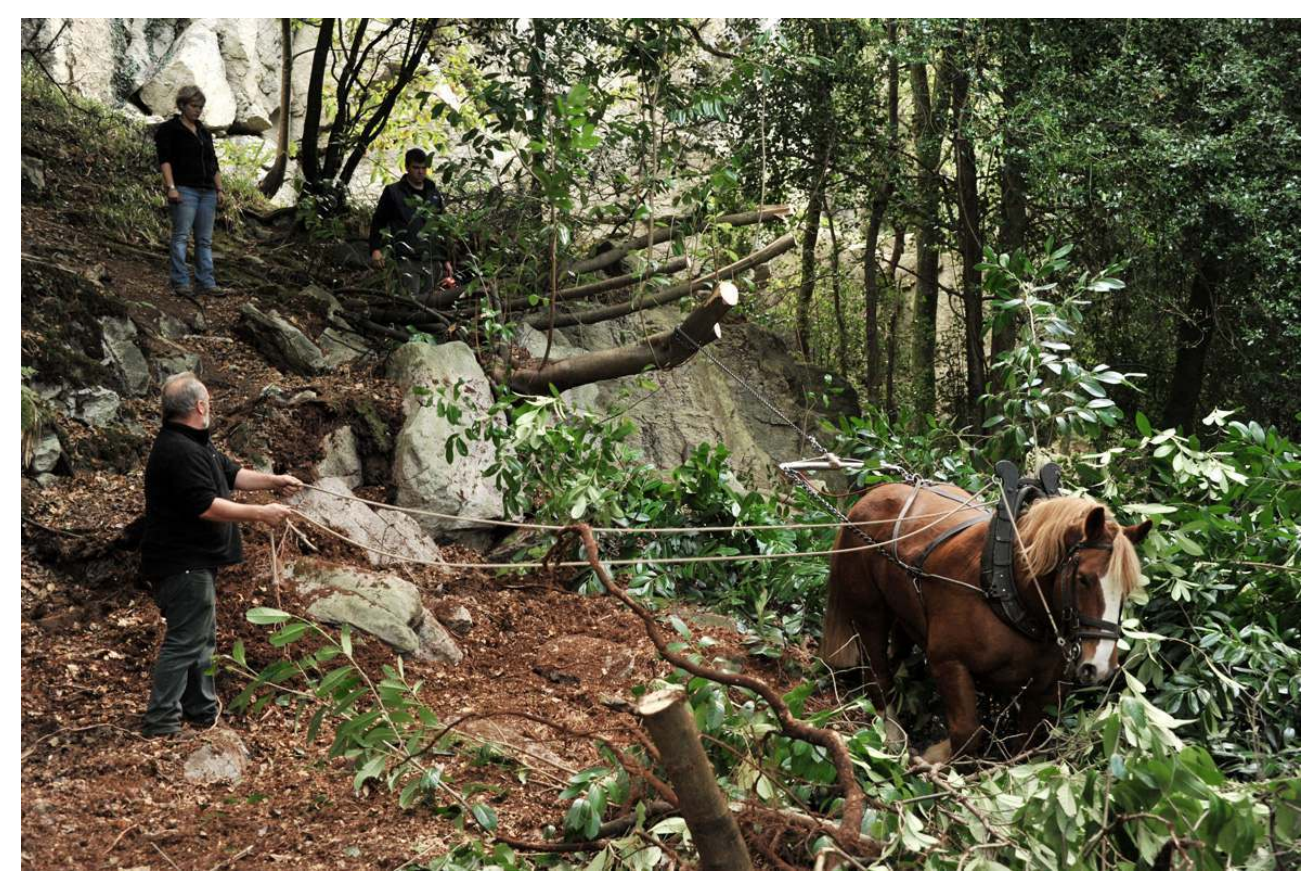

Jean-Jacques et Vincent Seïté au dessouchage de lauriers palme avec Oscar, au pied du Rocher de I'Impératrice sur la commune de Plougastel (Finistère).

Phot. Dugast, Jean-Léo. (c) Jean-Léo Dugast.

8 Un autre chantier, tout aussi expérimental, s'est ouvert en 2010 avec les mêmes donneurs d'ordre (conseils généraux et ONF) : il fallait arracher des bâches de plastique posées quelques années plus tôt pour protéger des plantations effectuées dans les zones de captage des eaux potables (en Bretagne c'est un vrai problème). Aucun outillage n'était prévu et l'arrachage du plastique lui-même n'avait pas été non plus envisagé. Ils ont testé une décavaillonneuse (charrue utilisée dans les vignes), ça marchait mais c'était trop dur à cause des racines. Ils ont alors créé un prototype en bricolant des pièces empruntées à des machines différentes.

9 Ce qu'ils aiment particulièrement dans leur métier, c'est trouver des solutions pour les problèmes que leur soumettent leurs différents commanditaires, et chercher des débouchés, diversifier, à partir du pur travail de débardage de bois. Un exemple de commande municipale: l'installation au printemps et l'extraction en automne d'un 
escalier de bois $(850 \mathrm{~kg})$ dans les dunes protégées de Beg Meil, la célèbre plage de Bénodet, dans le Finistère sud.

Pour eux, les qualités d'un cheval de travail sont la générosité et le calme. Qu'est-ce que la générosité ? «Il doit donner plus qu'il ne peut, quand on a besoin ». Précision : « Mais attention, il ne faut pas lui demander trop souvent ». Ils ont appris à doser et ils sont très attachés à leurs chevaux. Aucun ne part à la boucherie. Les débuts étaient plus tendus, travail en semaine et compétition le week-end, l'écurie est importante à présent, les chevaux peuvent respirer d'un chantier à l'autre et le sport est réservé à des spécialistes. La deuxième qualité d'un cheval de travail est donc le calme. Celui qui en est pourvu est «facile à gérer dans ses efforts », il sait se reposer et récupérer, attendre tout seul, rester tranquille. Bien utilisé, il peut durer quatorze ans en très bonne forme, après quoi il diminue (fig. 4).

Figure 4

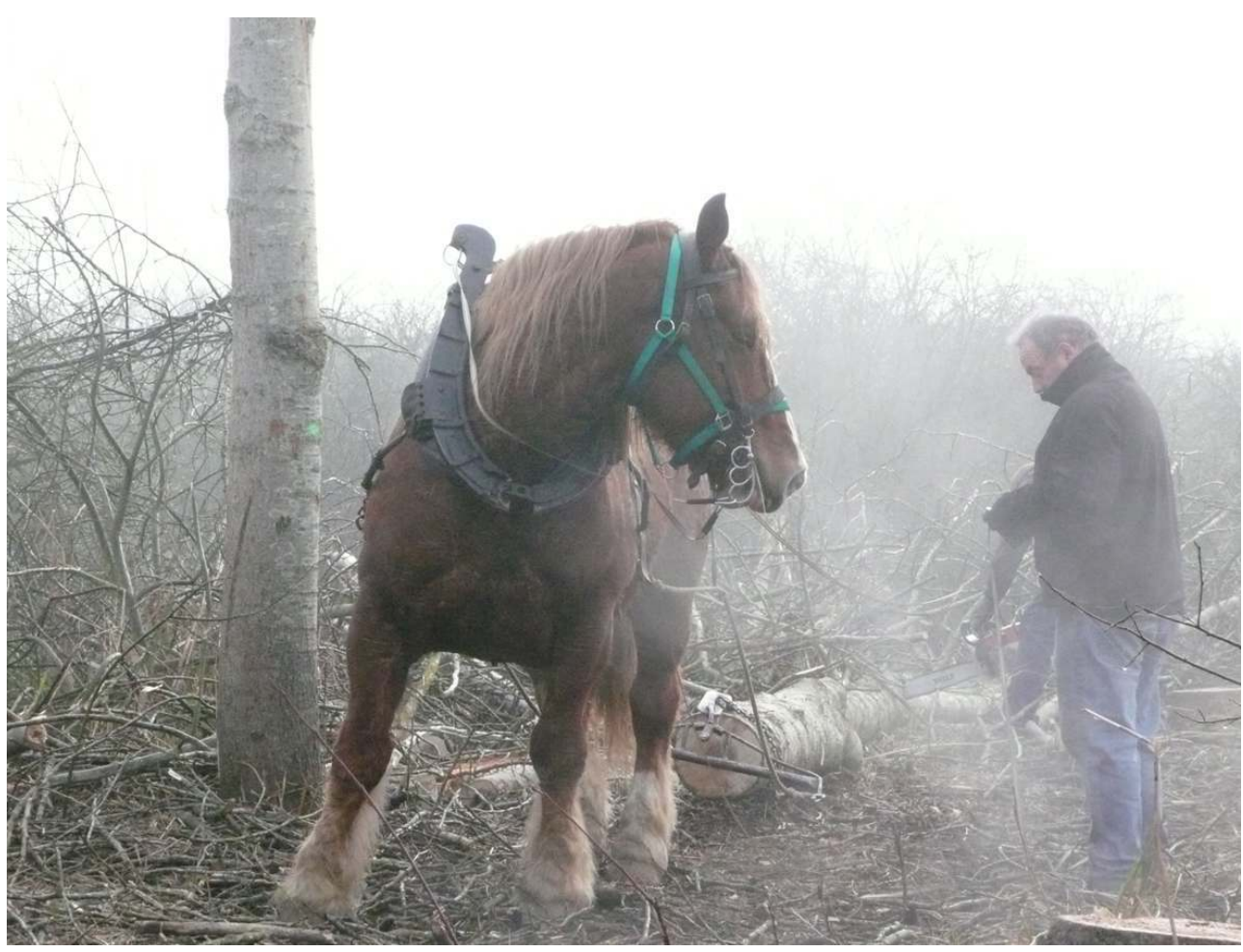

Repos et connivence au Marais de Larchant (Seine-et-Marne).

Phot. Lizet, Bernadette. (C) Bernadette Lizet.

11 Mettre les jeunes en route, c'est difficile, surtout les dessoucheurs qui doivent avoir du caractère. Il faut un an et demi à deux ans pour fabriquer un partenaire sûr et facile, qui ne panique quand il est coincé et ne s'épuise pas inutilement, car cette activité-là est très fatigante. Description des débuts de leur spécialiste d'avenir, Ubert: «On a galéré pendant sept-huit mois, il était très nerveux sur le gros bois, aujourd'hui il est agréable, il fait confiance, il sait que les hommes qui le font travailler vont trouver une solution quand ça se corse ». On ne recherche pas la même chose en dessouchage et en débardage :

On sait qu'ils lâcheront pas, et en breton, on en trouve plus facilement que dans d'autres races... Le savoir-faire des hommes n'est pas le problème, dessoucher ce n'est pas compliqué, le plus compliqué c'est d'avoir de bons chevaux. Sur dix qu'on 
va essayer, un va sortir bon. On arrive à le détecter en débardage, pas rapport à son comportement.

chantiers. Commentaire sur les conditions en forêt cet hiver 2013: "Dans la boue jusqu'aux genoux pendant trois mois, tout le monde était très fatigué... Il faut protéger le cheval, savoir faire ce qu'il faut pour qu'il soit capable de travailler le lendemain, même s'il a travaillé dans la boue toute la journée » (fig. 5). Pour bien faire travailler, il faut du bon matériel. Le collier est l'objet de toutes les attentions. Son perfectionnement va de pair avec le souci du confort (éviter l'échauffement et les blessures d'encolure) et l'amélioration de la performance, qui conditionne la rentabilité. On remarque une circulation des colliers dans le temps (objets traditionnels, repris aujourd'hui sur certains points stratégiques) et dans l'espace (de la Belgique, terre de débardeurs, à la France).

\section{Figure 5}

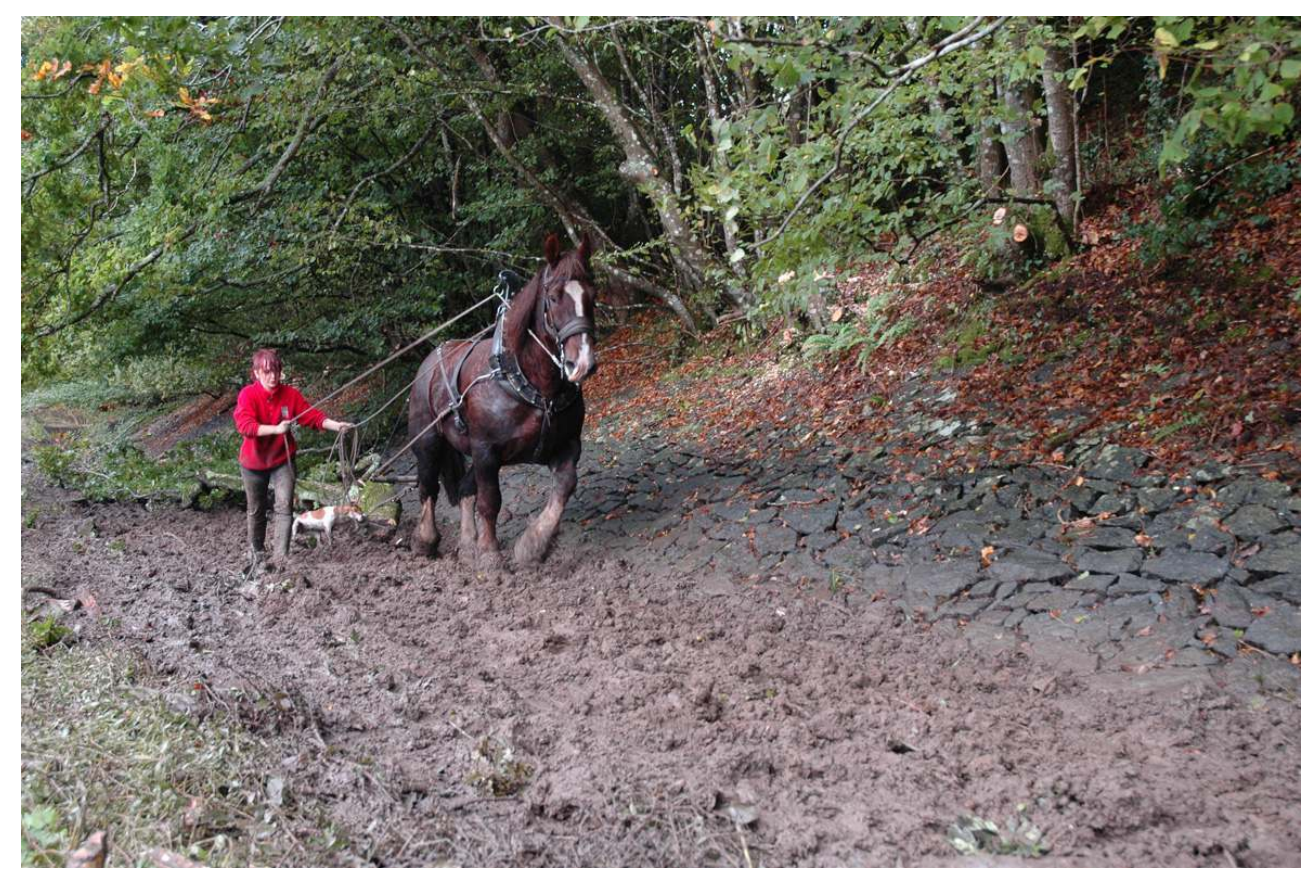

Emma Piton, stagiaire chez les Seïté, à la manœuvre dans un débardage pour la SNCF, le long de l'Élorn (Finistère). C'est marée haute, le travail se fait dans la boue.

Phot. Seïté, Vincent. () Vincent Seïté.

14 Vincent Seïté dit modestement que « la base c'est toujours pareil, un cheval ça tire avec les épaules ", mais il n'en reste pas moins que l'activité de débardage-dessouchage est innovante sur tous les plans, l'outillage, la relation au cheval, la relation aux donneurs d'ordre, la relation à l'environnement; le père et le fils Seïté sont devenus des naturalistes de terrain, ils sont incollables sur la biologie du laurier palme. Ils ont une conscience aiguë d'être héritiers d'un très ancien savoir-faire. Une compétence d'éleveur (la race bretonne avec ses singularités, qualités et défauts), de dresseur et d'utilisateur de la force des chevaux. Mais face aux problèmes qui surgissent sur chacun des chantiers, l'héritage culturel est sans cesse perfectionné, de la race aux outils, en passant par la technique de dressage et d'utilisation. Le respect de la nature et de la vie est une forte 
composante de la culture professionnelle: «L'écologie est le premier argument de la traction animale », dit Vincent.

\section{Utiliser en montagne}

15 Deny Fady arrive aux chevaux de travail « par l'arrière, c'est-à-dire par le matériel qu'ils tractent ». Il souligne le fait, car de son point de vue «la plupart des utilisateurs y sont venus par le biais de leur intérêt pour le cheval en général ou de leur compétence en élevage ou en attelage ». On remarquera que le terme qu'il choisit pour parler de son activité marque cette époque de la deuxième relance du cheval de trait, «l'utilisation ». L'une des particularités de cette communauté réunie autour d'un nouveau cheval de trait qui n'est plus strictement agricole était la très forte hétérogénéité des trajectoires et des motivations, et notamment le goût pour l'attelage de loisir ou sportif'. Deny Fady n'a pas reçu de culture équestre et s'il se souvient de son grand-oncle qui labourait la terre de la famille près de Saint-Marcellin en Isère avec deux mulets liés par un joug de colliers ${ }^{7}$, il a dû tout apprendre par la suite.

Après un bac général et technologique, de courtes études de biologie et un passage par l'école normale (lieu de formation des instituteurs à l'époque), il quitte rapidement l'Éducation nationale : il a un projet d'école rurale parallèle organisée autour d'une ferme vivrière. En 1979, il a 21 ans. Il s'installe en communauté agricole dans un hameau savoyard à 1400 mètres d'altitude, dans l'optique de vivre en autonomie et de faire avancer son projet. Deux ans plus tard, la communauté se disperse et il construit une petite ferme avec Odile, sa femme. Ils louent 12 ha à l'Association foncière pastorale (AFP) de la commune. Odile s'installe "jeune agricultrice» déclarée à la Mutuelle sociale agricole (MSA) et lui-même réintègre l'Éducation nationale à temps partiel comme enseignant de technologie dans un lycée agricole : il devient double actif. Sur la ferme d'Églantine aujourd'hui, il y a deux vaches tarines (race savoyarde) qui produisent chacune un veau élevé à cent jours, deux cochons à l'engraissement, un cheval de trait de race comtoise, une ânesse (croisée baudet du Poitou) et une grande basse-cour. Ils obtiennent une gamme complète de produits laitiers, de charcuterie, de confitures et de légumes pour la consommation familiale (ils ont trois enfants). L'activité est complétée depuis 2013 par un accueil à la ferme, yourte et table d'hôtes. Ruby le cheval comtois assure tous les transports. Il rentre 15 ha de foin coupé à la motofaucheuse, fané et mis " en trousses 8 » à la main. Il débusque ${ }^{9}$ et débarde le bois de chauffage et de construction, ramène le petit lait destiné aux cochons depuis la chèvrerie voisine. Il laboure aussi environ un hectare de jardins, parcelles familiales et collectives (choux à choucroute, potimarron, pommes de terre et légumes courants) et accomplit divers travaux « de cour 10 \%. Il tire enfin la roulotte durant les vacances. Églantine l'ânesse est mise à contribution pour divers transports dans le village, elle ramène le bois en bûches pour le four à pain, elle sarcle le jardin et accompagne des randonneurs.

En 1982, Deny Fady réalise son premier chantier pour l'ONF : il doit transporter des plants de mélèze, des piquets et des rouleaux de barbelés, qui vont permettre de créer une forêt de protection contre l'érosion sur un ancien alpage d'altitude. Il conçoit une petite carriole, toute en aluminium. Elle sera tirée par Coco, qui travaille sur la ferme communautaire. Ce petit cheval «de pays ${ }^{11}$ » a été échangé à un maquignon devant l'abattoir, contre quatre chevreaux. Coco était têtu, mordeur, mais courageux et tout le village venait l'emprunter pour labourer les jardins. Pour réaliser la carriole, dont le 
plancher doit être très bas, Deny s'inspire de la petite voiture porte-bidons traditionnelle des alpages savoyards. Son prototype a du succès (fig. 6), on lui demande d'en diffuser les plans dans Alternatives Paysannes, le bulletin des Comités d'études et de propositions pour le développement des activités paysannes (CEP) ${ }^{12}$. Il fait alors partie d'un groupe de réflexion sur la "mécanisation adaptée » avec les CEP et la Compagnie d'études industrielles et d'aménagement du territoire (CINAM) de Maurice Ogier, un inventeur hors pair, qui avait une grande expérience en matière de construction de tracteurs et autres matériels conçus avec des technologies mécaniques minimalistes, adaptées aux pays en voie de développement. L'idée germe, au CEP Savoie, de créer un groupe "traction animale", Deny Fady s'implique immédiatement. Les membres du groupe entrent en relation avec une dizaine d'utilisateurs actuels ou anciens, des paysans pour la plupart, issus de la région Rhône-Alpes toute entière. Ils rencontrent le fabriquant d'outils Jean Nolle ${ }^{13}$, qui les intéresse parce qu'il travaille dans des ateliers dont l'équipement est très simple. Mais il leur faut des véhicules équipés de freins en montagne et il va s'avérer que Jean Nolle ne croit pas à la recherche accomplie par des utilisateurs.

\section{Figure 6}

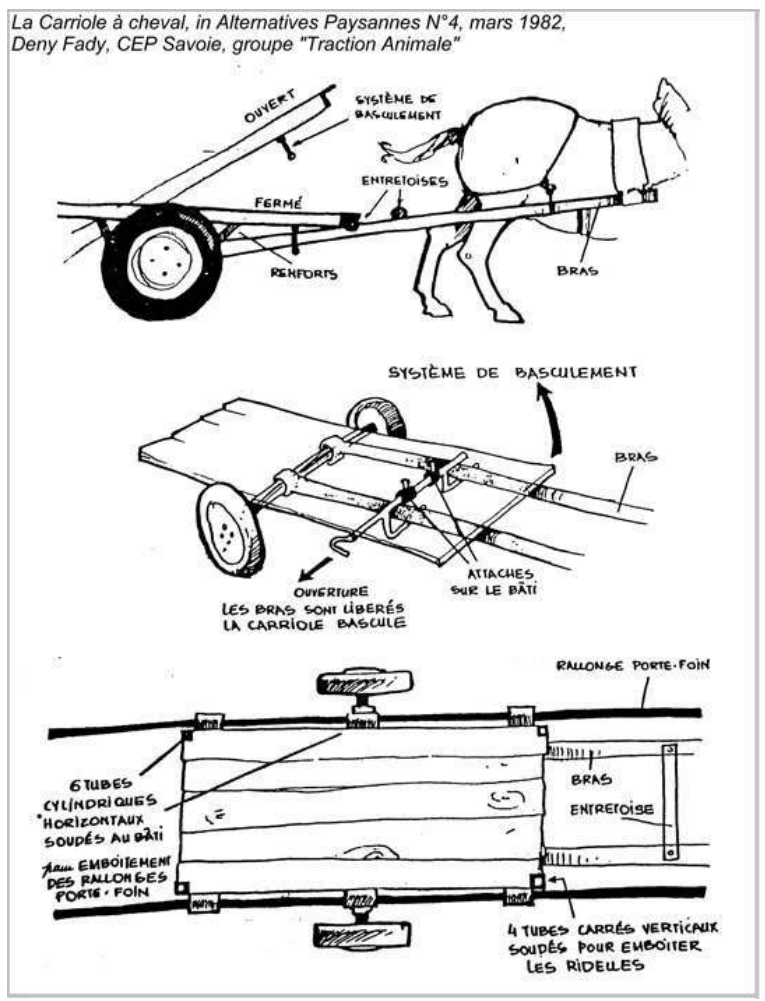

Plan de la carriole moderne, réinterprétant une petite voiture traditionnellement utilisée en alpage.

Phot. Hippotèse. (c) Hippotèse.

Avec la collaboration du centre de formation professionnelle agricole (CFPPA) du lycée agricole de La Motte-Servolex en Savoie, ils se lancent et élaborent leur propre porteoutils, «l'ATSOU » (Avant-train support d'outils universel) et aussi leurs premiers harnais en sangle, ainsi que des arceaux de traction, sur un modèle traditionnel de la maison Taravello de Romans-sur-Isère. Ils diffusent le résultat de leurs recherches sous forme de 
fiches techniques et organisent des stages d'auto-construction de ces matériels: les utilisateurs fabriquent ce dont ils ont besoin.

Plusieurs prototypes d'ATSOU voient le jour en 1984. Cette même année, ils étudient les colliers de type norvégien sous toutes leurs coutures, en construisent deux modèles (bois et métal) avec un bourrelier local. Le groupe est alors en quête de solutions pour la construction artisanale, voire l'auto-construction de colliers de travail confortables pour le cheval, efficaces et réglables.

En 1985, Deny Fady et son groupe participent à une enquête sur la situation de la traction animale en France, financée par le ministère de l'Agriculture ${ }^{14}$. L'année suivante, une rencontre décisive a lieu avec le groupe Franche-Comté de l'association de recherche sur la traction animale et le portage (ARTAP), créée par Jean-Jacques Marquard ${ }^{15}$. Jean-Louis Cannelle, l'animateur de ce groupe franc-comtois, est un grand connaisseur des chevaux de trait. Paysan (membre actif de la Confédération paysanne) et éleveur de comtois, il joue un rôle moteur dans le syndicat de race et, en pleine période d'orientation bouchère, il pèse de tout son poids pour opérer un retour du modèle vers un cheval d'utilisation. Il fait travailler toutes ses juments reproductrices sur la ferme familiale (labour, exploitation du bois, transport du lait). Le Savoyard et le Franc-comtois ont la même philosophie : il faut des actions concrètes pour faire évoluer le cheval de travail et son équipement. En 1986, ils créent l'association Hippotese (association hippomobile de technologie et d'expérimentation du Sud-Est) qui publie les premiers Cahiers techniques ${ }^{16}$ sur le matériel et les harnais, une information pratique et pédagogique. Puis viendra Hippobulle, le bulletin très illustré de l'association. Des stages d'initiation à l'utilisation des chevaux de travail sont organisés deux fois par an sur la ferme de Jean-Louis Cannelle en Franche-Comté. Qu'est-ce qu'un cheval de travail dans ce réseau? C'est un cheval "d'attelage ", " permettant de pratiquer en toute sécurité des activités à l'allure du pas, en milieu agricole, forestier ou urbain » (fig. 7).

Figure 7

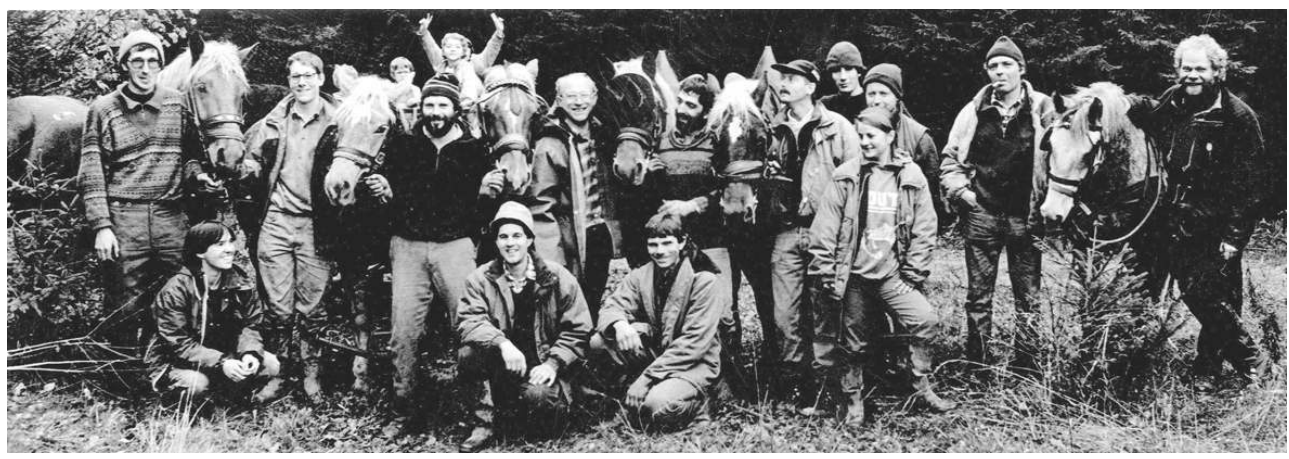

Premier stage organisé par Hippotèse, en 1983.

Phot. Hippotèse. (c) Hippotèse.

21 À partir de 1991, un voyage d'étude est programmé presque chaque année à travers l'Europe, Allemagne, Belgique, Grande-Bretagne, Pologne, Suède, Italie, Autriche... Un nouveau prototype d'avant-train voit le jour en 1995, le Cabri (à trois roues, dont une directrice) ; il sera le premier d'une série d'avant-trains spécialisés, agricoles et forestiers. L'association Hippotese s'approprie l'outil informatique: création d'un site web pour diffuser les résultats des recherches à moindre coût (1998), puis d'un blog (2005), qui diffuse un billet par semaine sur le cheval de travail. En 1999, création du Centre 
européen de ressources et de recherches en traction animale (CERRTA) ${ }^{17}$. C'est le «bras armé » (de bonnes intentions, mais tout de même financées!) d'Hippotese, qui reste « l'aile politique ». Le CERRTA joue le rôle d'un bureau d'études sur des projets en traction animale. Il emploie trois salariés pour répondre à des demandes d'intervention technique, contre rémunération. L'association Hippotese, qui compte environ 300 adhérents en 2014, reste un lieu de réflexion et d'expérimentation, de mise en commun des pratiques. Elle tient à sa liberté de ton et diffuse tous ses résultats de recherche en licence libre ${ }^{18}$. Elle fonctionne uniquement avec des bénévoles et sans subvention (fig. 8, fig. 9).

\section{Figure 8}

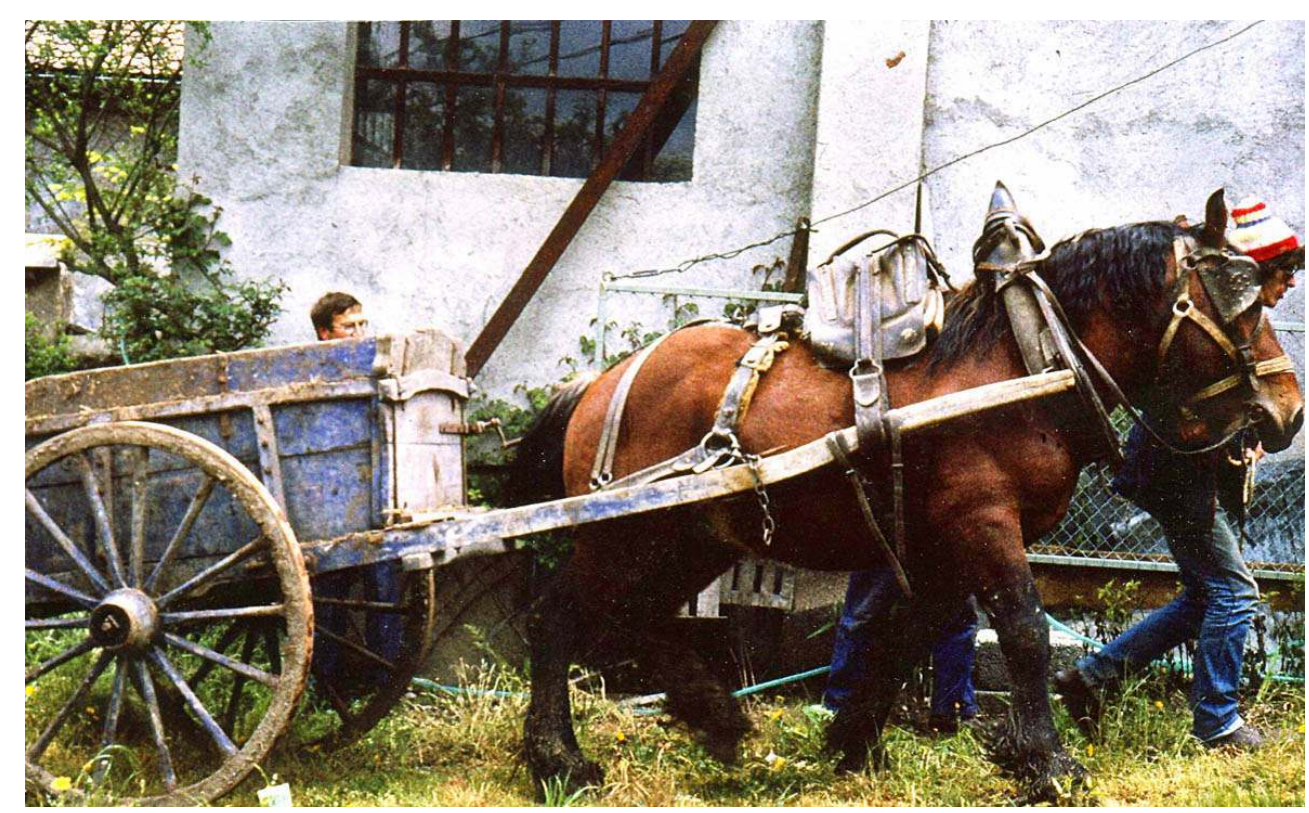

Un trait d'union entre la traction animale paysanne et néo-rurale. Activité du groupe « Traction animale » du CEP Savoie en avril 1984.

Phot. Hippotèse. (c) Hippotèse. 


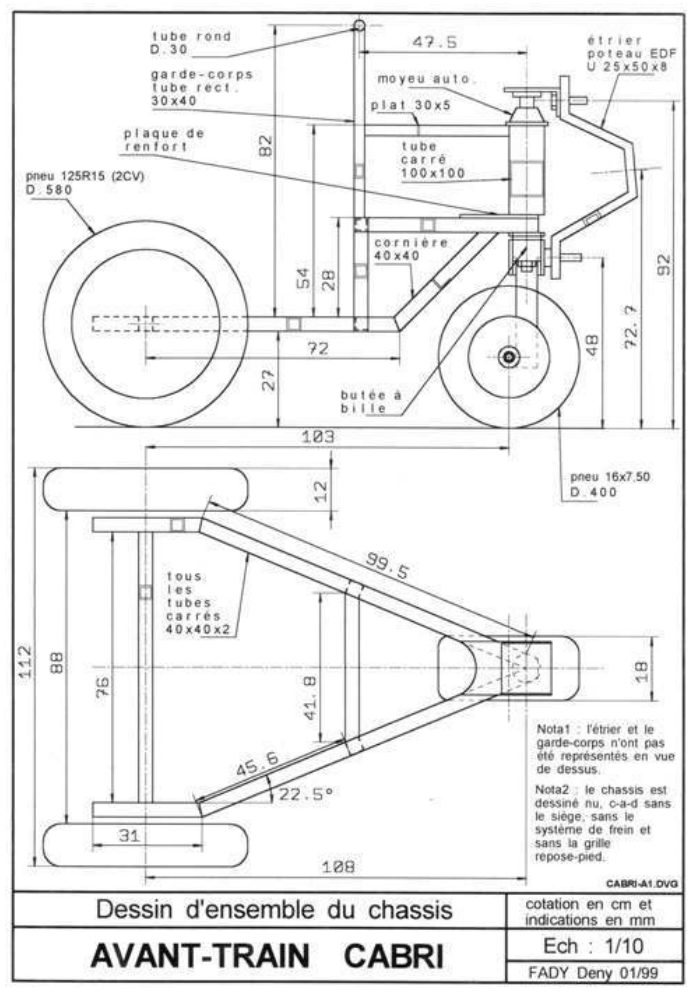

Le Cabri, prototype d'avant-train, dont la première version a été inventée en 1994.

Phot. Hippotèse. (c) Hippotèse.

\section{Labourer les vignes en Bordelais}

Pour Ramon Garcia, le mouvement de la terre qui se retourne sous le versoir est symbolique, et c'est encore plus vrai lors de la préparation du sol avant un semis de céréales. Le labour marque le début du calendrier cultural d'une terre nourricière et dans les vignes, il permet d'enfouir le couvert végétal avant le passage des griffes, puis de la décavaillonneuse. C'est l'acte premier, et c'est donc avec détermination que Ramon Garcia choisit ce terme pour présenter le métier auquel il a choisi de revenir. Il est en effet fils et petit-fils de laboureur dans les vignes. Paysan dans la Mancha d'Albacete en Castille, son grand-père travaillait ses propres terres avec des mules et il vendait ensuite le raisin. Il lui arrivait de s'embaucher en complément dans de grandes fermes. Le père immigre en France en 1961, près de Bordeaux, à Saint-Avit-de-Soulège. Il prend une ferme en métayage, dix hectares de vigne et huit de prairies, qui nourrissent les douze vaches laitières (des « hollandaises ») et la jument (de race indéterminée, et qui ne pouline pas). Il accomplit sa dernière campagne de labour en 1968 et devient alors salarié (chef de culture). Quant à Ramon, il se lance dans la vie active dans une toute autre optique. Après l'école hôtelière à Bordeaux (bac "pro») et un long séjour aux Antilles, il devient chef cuisinier d'un général à Bordeaux, puis émigre en Australie, revient et intègre un peu par hasard le lycée viticole de Monbazillac (Dordogne) pour suivre un brevet professionnel agricole. Il s'implique avec sa compagne dans la gestion d'un domaine acheté dans le Bergeracois par le P.D.G. d'une compagnie pétrolière russe, le reconvertissent en «bio ». 
Il achète alors Charly, un bardot (produit du croisement entre l'étalon et l'ânesse ${ }^{19}$ ) pour le travail, un choix qui s'avère totalement inadapté. Il est licencié en 2008 et va se former en Bourgogne chez un "prestataire de services », engagé dans l'agriculture biologique, qui «tourne » avec trois juments (deux percheronnes et une auxoise). Pour lui « c'est un schéma qui fonctionne », il va l'appliquer en Bordelais. En mars 2009, il crée sa propre société, Aequitaine, une entreprise unipersonnelle à responsabilité limitée (EURL) basée à Eynesse en Gironde, entre Bergerac et Bordeaux. Il achète Olympe à un agriculteur céréalier du Lot-et-Garonne qui élève aussi des canards (25000 par an), et des chevaux bretons. Il possède cinq poulinières, participe aux concours de race et conserve un ou deux poulains chaque année, qu'il dresse en faisant son jardin et en attelant à une voiture de promenade. Olympe a six ans lorsqu'elle découvre la vigne avec Ramon mais elle est patiente, calme et appliquée, ce qui permet d'emblée au laboureur encore novice de se concentrer sur le réglage de son outil, charrue ou décavaillonneuse (fig. 10).

Figure 10

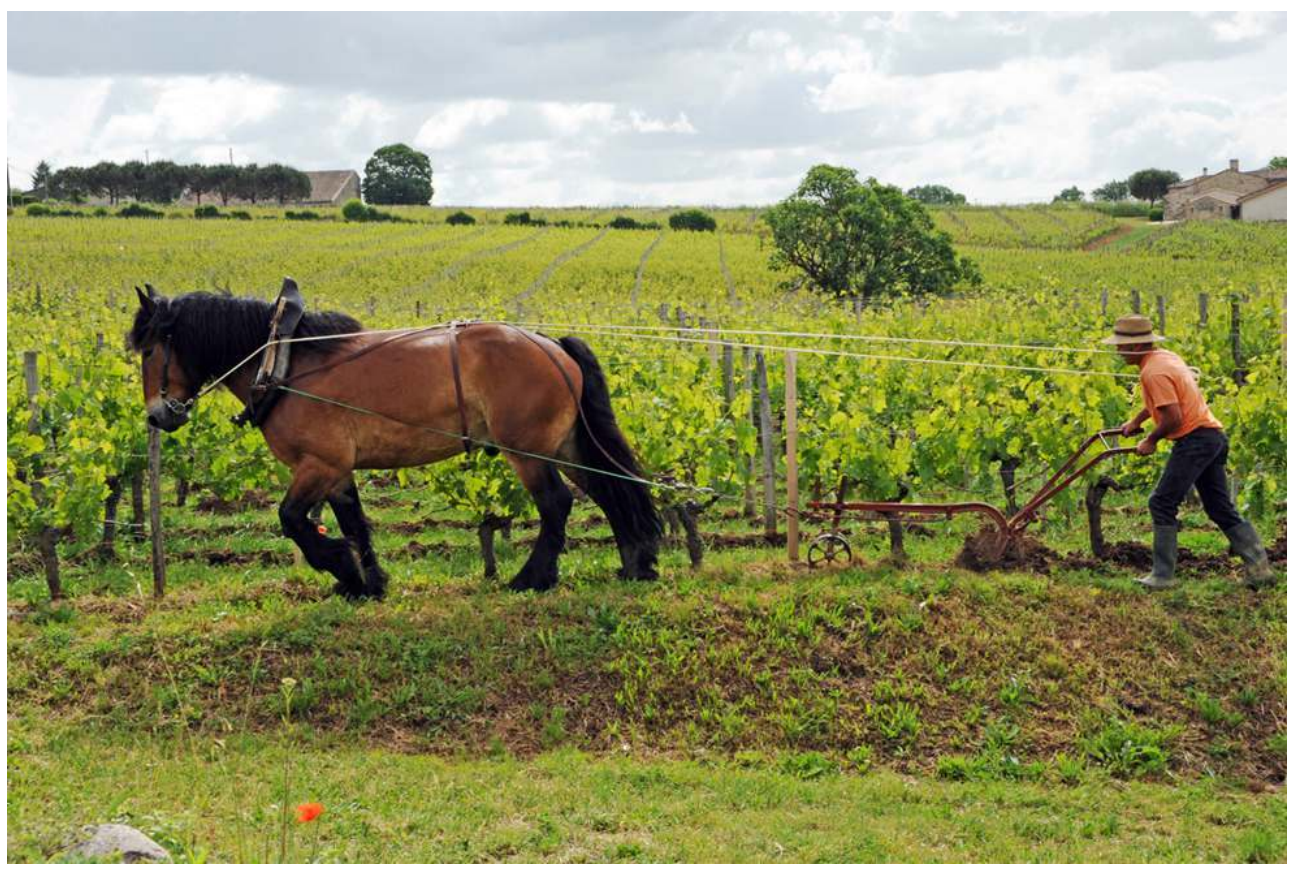

Ramon Garcia «chausse » (buttage de printemps) avec Kalin, trait ardennais, sur le domaine Le Prieuré en appellation Saint-Émilion (Gironde, juin 2015).

Phot. Dugast, Jean-Léo. (c) Jean-Léo Dugast.

Il comprend vite que le travail du sol est lent en traction animale : il faut pouvoir réunir plusieurs équipages sur une même parcelle. De mars 2009 à janvier 2011, il monte une écurie de travail, vingt-deux chevaux, douze juments et dix hongres, pour l'essentiel des "trait breton", achetés en Aquitaine (chez trois éleveurs différents), qu'il confie en dressage à l'éleveur d'Olympe. Ramon les met ensuite à la vigne. Au début, il achetait des bêtes en âge de travailler (plus de trois ans), puis il a recherché des poulinières (plus de douze ans). Il écarte ainsi de la boucherie 18 chevaux de travail. Depuis 2012, il sélectionne ses propres poulinières, fait naître des poulains (un à trois par an), ce qui suffit pour la rotation : les animaux qui ont accompli deux ou trois campagnes ont pris de la valeur, il les vend. Son écurie de travail passe l'hiver à Eynesse, dans les quinze hectares de prairies vallonnées, des terres «porteuses» sur calcaire affleurant. Une 
grange de $500 \mathrm{~m}^{2}$ abrite le fourrage et les animaux qui ont besoin de soins quotidiens particuliers (fig. 11).

Figure 11

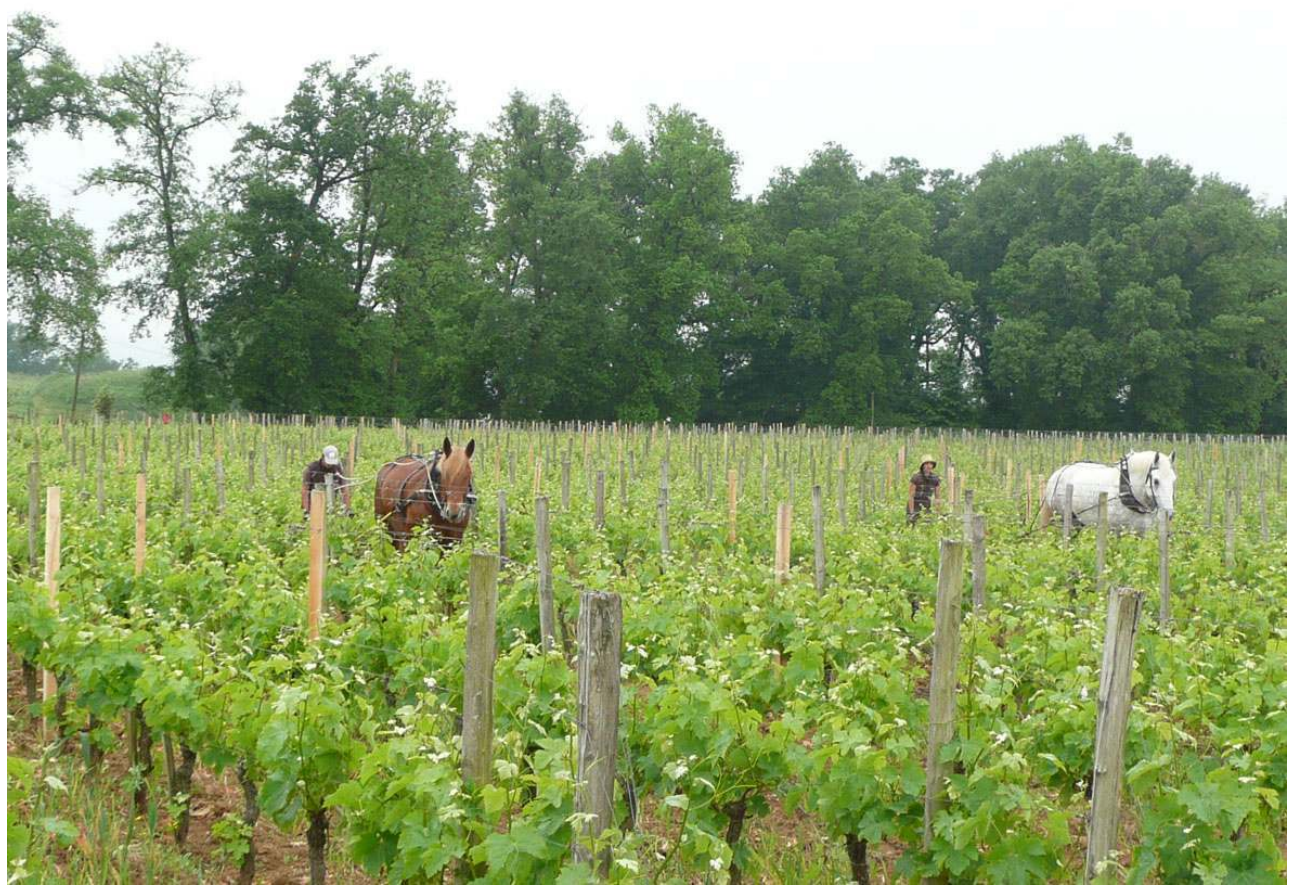

Ode et Sébastien Mizier (prestataires) à Château Troplong Mondot avec Ulysse (percheron gris) et Violette (breton-comtoise), appellation Saint-Émilion, 12 mai 2015.

Phot. Lizet, Bernadette. (c) Bernadette Lizet.

Pendant la campagne de labour (mars à novembre), les chevaux séjournent dans les six domaines viticoles clients d'Aequitaine, situés dans le Bordelais et le Bergeracois, en Lotet-Garonne également. De la petite propriété (moins de $10 \mathrm{ha}$ ) qui commercialise son vin à moins de $10 €$ au premier grand cru classé ( $1500 €$ la bouteille), la gamme est très ouverte. Pour tous, la motivation à passer au cheval est d'abord agronomique : les sols doivent être préservés. Quelques domaines n'ont pas de prairie, il faut alors installer un paddock qui accueillera les chevaux pendant la durée du chantier. Il faut aussi transporter les chevaux, le foin et le complément de céréales avec la bétaillère et le van ; l'eau est fournie par le château. Pas d'hébergement en boxe en campagne de labour, ce qui présente l'inconvénient d'allonger le temps de pansage .

Pour éviter de passer trop de temps sur la route et rester à proximité des chevaux, Ramon loue parfois un gîte à proximité des domaines. Mais il est fréquent qu'un salarié, ou le propriétaire lui-même, décide de veiller sur les animaux. Impliquer les personnes sur place est important, car la relation change : il ne s'agit pas d'une simple prestation, mais d'un échange. C'est particulièrement vrai lorsqu'un poulinage survient. En 2012, trois bretonnes ont mis bas à Château Le Puy, Château Latour et Château Couhins-INRA. Installées dans un petit paddock avec une tente équipée d'une lampe à la sortie pour intervenir rapidement, elles étaient surveillées de très près. Surveiller et assister, tout le monde s'y est mis. Le nom des poulains a été choisi par les salariés des domaines et lors de la veille pour la naissance de Cannelle à Château Le Puy, le propriétaire a ouvert une 
bonne bouteille de 1999 (sans soufre) : un grand moment ! Cahouet de Latour a été admiré et caressé par l'ensemble du personnel et le fondateur d'Aequitaine espère bien le voir un jour labourer les parcelles du domaine. Un «tracteur » est né à Latour! Les poulinages ramènent la vie dans les vignobles et créent du lien.

L'entreprise est aujourd'hui équipée de six porte-outils pour griffer, quatre charrues vigneronnes, trois décavaillonneuses Rotex, une double décavaillonneuse Horse One (nouvelle génération), six canadiennes (trois, cinq, sept et neuf dents), trois traîneaux (pour répandre le compost, transporter les piquets, etc.). Il y a aussi un pulvérisateur électrique "trois mètres hors tout» et un autre de deux mètres, une poudreuse " entraînement par les roues ". Cinq nouveaux outils ont fait leur apparition sur le marché en 2013, tous français (Horse one, Équinoxe, Tech trait, Mata vigne, Pégase) et beaucoup trop lourds. Les anciens, notamment ceux qui ont été construits au début des années 1970, restent opérationnels, comme la très vieille charrue Millon et aussi l'Universala de chez Ebra, la décavaillonneuse Rotex qui fait l'unanimité auprès des professionnels du travail du sol avec le cheval. La tarière (pour retirer les racines du cep mort) équipée d'un moteur thermique est attendue avec impatience. Elle permettra de travailler durant l'hiver (fig. 12).

\section{Figure 12}

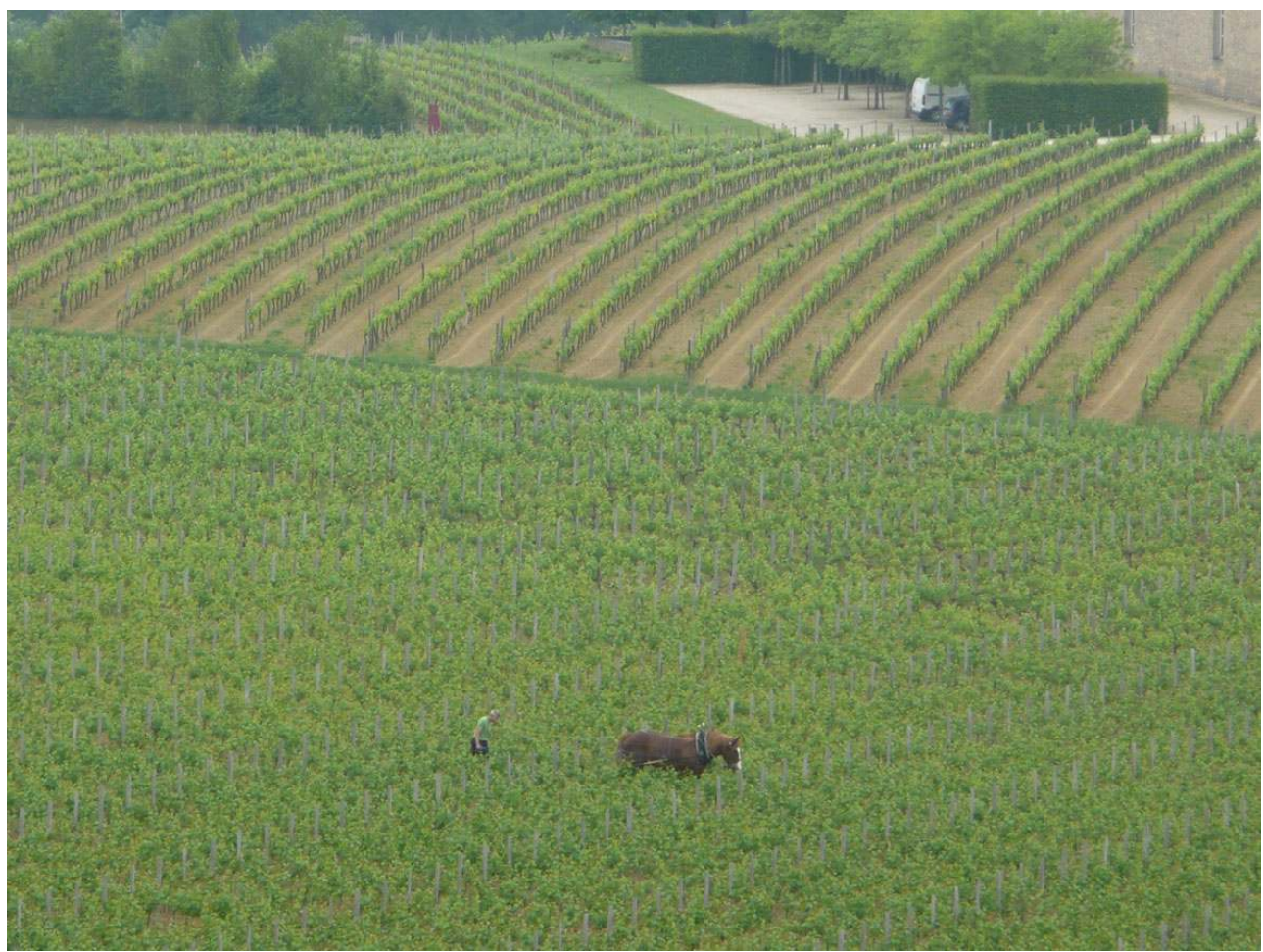

Jérôme Vos avec Nounours (breton) à Château Faugères, Saint-Émilion, 12 mai 2015.

Phot. Lizet, Bernadette. (c) Bernadette Lizet.

Côté harnais, comme en débardage, le collier constitue la pièce la plus importante. S'il n'est pas adapté il peut blesser, ou diminuer le rendement en termes de transmission de force. Dans la vigne, le cheval accomplit un travail régulier, d'endurance, mais l'effort peut être très important avec ces outils modernes, surtout à l'automne et au printemps, lorsque les terres sont lourdes. Aequitaine a démarré avec deux colliers amish que son formateur bourguignon utilisait. Puis il a adopté un «Bernard Lacuche» (bourrelier- 
sellier dans le Lot), deux «Jean-Pierre Bontemps» (Périgueux), car "développer l'économie locale, c'est important ». De vieux colliers ont également été achetés chez un vigneron proche de Sainte-Foy-la-Grande, éleveur de percherons ; aujourd'hui retraité, il se passionne pour ces matériels et les restaure. Le collier qui fait référence aujourd'hui est français. C'est l'« AFH » (atelier fabrication harnachement) d'une bourrelière établie en Haute-Vienne, Stéphanie Boudin, qui a repris le brevet de Camille Junien (constructeur de matériel de traction animale dans les années 1980), le fameux « CJ", polyvalent et réglable. Depuis un an, Stéphanie Boudin commercialise une version «vigne», plus légère.

L'adéquation du gabarit et du tempérament du cheval au travail accompli est une question centrale. Olympe est le type même du cheval vigneron, à l'écoute de la moindre résistance que la décavaillonneuse peut rencontrer. La jument règle ses gestes d'ellemême, tout en finesse, au plus près des sensations qu'elle reçoit dans le collier : «Elle s'arrête avant que le laboureur ne ressente la racine du cep sur les mancherons ». Elle toise $1,62 \mathrm{~m}$ et pèse environ $800 \mathrm{~kg}$, un format que Ramon considérait comme optimal pour son activité. Mais l'arrivée sur le marché de nouveaux outils issus d'une cinquantaine d'années de " néo-mécanisation » en traction animale le fait changer d'avis ${ }^{20}$. Les doubles décavaillonneuses («DK» pour les connaisseurs) permettent de traiter deux demi-rangs et doubler la vitesse (trois kilomètres à l'heure). Elles demandent évidemment plus d'efforts que les traditionnelles, imposent des chevaux plus lourds. Pénélope, une boulonnaise de $900 \mathrm{~kg}$, a rejoint l'équipe en mars 2014 et elle a tout de suite « donné le sentiment de se promener avec cette double DK Horse one ». Ramon précise : «Disons qu'après trois heures de travail, elle n'était pas épuisée, moi si ! ». L'intégration de Pénélope, spécialiste de la «DK» a été de pair avec une diversification des races au travail utilisées dans l'entreprise. À la fin de l'année 2014, le panel est presque complet : deux ardennais, un cob normand (« un peu juste» quant au gabarit), des comtois, deux percheronnes et un trait du Nord. Seul l'auxois fait encore défaut. Explication : « On nous demande souvent s'il y a une race plus adaptée et n'ayant pas la réponse à ce jour, je veux comprendre si tel est le cas. Une autre raison est la possibilité pour les stagiaires de découvrir dans un même lieu les neuf races françaises ».

Une telle écurie de travail et un tel parc de matériel en traction animale ne sont plus chose fréquente en France. Ce développement a été de pair avec une forte dynamique de formation. Dès son installation, Ramon Garcia a reçu des demandes d'accueil de stagiaires, désireux de découvrir le métier de laboureur. Il a tout de suite répondu favorablement parce qu'il croit à la réintroduction du cheval dans les vignes et qu'il entend y contribuer activement. Il a dans l'idée de former des professionnels et de les aider à trouver leur place chez les propriétaires de vignobles, qui feraient alors l'acquisition des chevaux, les installant dans leur domaine. Il a ainsi initié quelque quatrevingt-dix personnes à la charrue, à la griffe et à la décavaillonneuse traditionnelle (la moitié seulement !). Une action très précise à réaliser autour des ceps, qu'il faut d'autant plus respecter que ce retour du labour animal s'effectue dans bon nombre de vignobles de haut rang (Latour, Pontet-Canet, Pape Clément, Smith Haut Lafitte, Le Puy, Le Prieuré, Troplong Mondot, Rocheyron, Peby Faugères, L'Escart...).

Ramon se souvient bien des deux premiers stagiaires qu'il a reçus en 2010: Didier, quarante-huit ans, ancien chef de culture dans les vignes, qui suivait une formation au CFPPA de Saint-Affrique dans l'Aveyron, et Jane, ingénieure " grande culture ", diplômée de l'école d'Angers, vingt-six ans, alors à la recherche d'un emploi. Il est ensuite sollicité 
par plusieurs centres de formation. En 2010, 2011 et 2013 c'est Agrobio 33 ${ }^{21}$, pour un cycle d'initiation de cinq jours. Trente personnes (trois groupes de dix) d'origine agricole et plus particulièrement viticole, propriétaires de vignobles en Aquitaine pour la plupart, des hommes et des femmes en parité. En 2010 et 2011 également, c'est Vini vitis bio ${ }^{22}$, en interne avec les salariés de Latour (une dizaine de personnes, des hommes en majorité). Il y aura aussi $X_{\text {perta }}{ }^{23}$ en 2012 et 2013: une vingtaine de salariés viticoles et de propriétaires, des hommes en majorité. Pôle emploi (2011 à 2014) lui a également adressé une trentaine de personnes, originaires d'Aquitaine, mais aussi de Paris, Orange, Marseille, Tarbes, Pau, Amboise, Normandie... Trente-cinq pour cent étaient des femmes. Depuis le printemps 2014, Aequitaine dispense par ailleurs une formation spécialisée pour des adultes en reconversion professionnelle au lycée d'enseignement général et technique agricole (LEGTA) de Blanquefort, près de Bordeaux. Dix stagiaires l'ont suivie (plusieurs chefs d'entreprise, une épouse de vigneron, deux BTS «viti-œno", des chercheurs d'emploi issus du tertiaire, en exacte parité hommes et femmes, tous d'origine locale) (fig. 13).

Figure 13

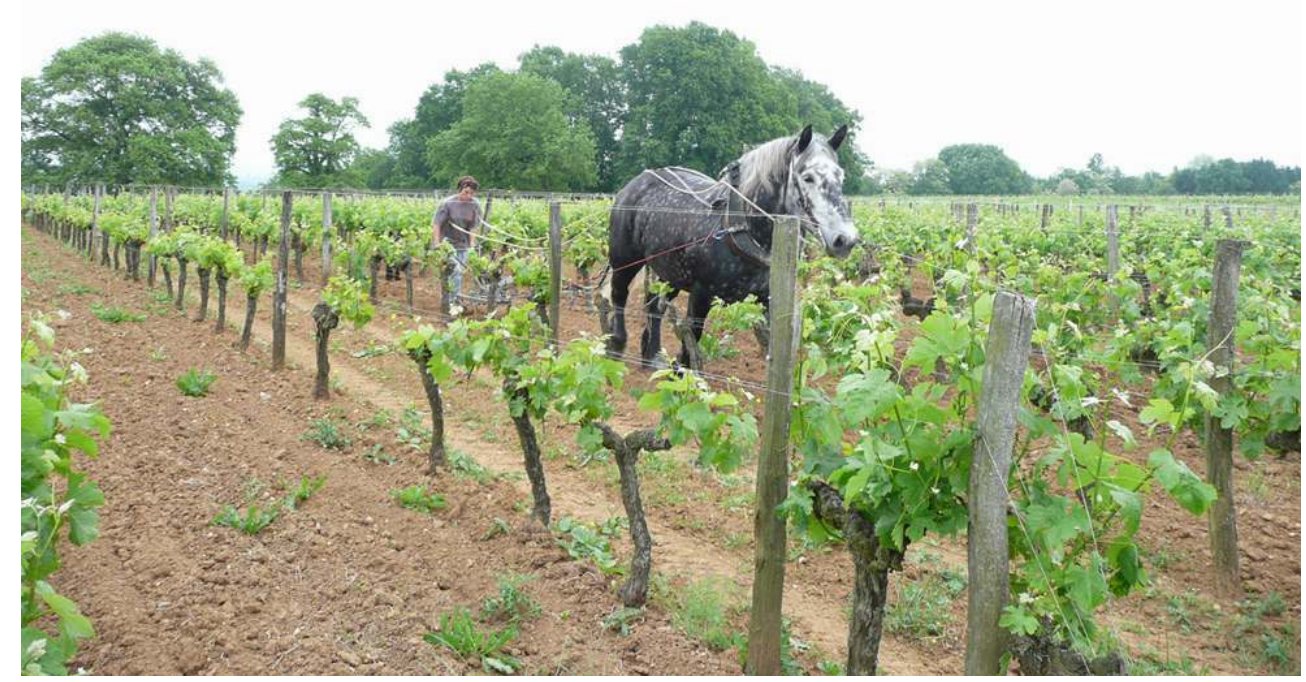

Anne-Sophie et Leila, Château Le Puy, Saint-Émilion, 12 mai 2015.

Phot. Lizet, Bernadette. ( ) Bernadette Lizet.

31 L'âge moyen est de 35 à 40 ans, mais les quinquagénaires sont bien plus nombreux que les jeunes de 20 ans. La motivation est de redonner du sens à la vie professionnelle. Les femmes sont très souvent des cavalières (ce n'est pas le cas pour les hommes) et les nouveaux outils peuvent leur poser problème; en «traditionnel », elles n'ont aucune difficulté. Les constructeurs en ont pris conscience et font des efforts pour faciliter l'utilisation. C'est avec intérêt que Ramon Garcia considère le rôle des femmes dans la réintroduction du cheval dans les vignes : 
Je suis convaincu de leur rôle dans la réintroduction du cheval, pas uniquement comme utilisatrices mais en tant que décideuses. Ayant connu le cheval dans leur jeunesse, les femmes qui occupent des postes de cadre, voire le statut de propriétaires de domaines, ont un rapport sensible avec le cheval, elles leur permettent de jouer une carte.

À peu près la moitié des stagiaires souhaite s'installer et intervenir en tant que "prestataire », l'autre moitié préfèrerait l'embauche (intégrer une équipe « en interne », chez le propriétaire). Ramon Garcia a gardé le contact, de près ou de loin, avec une soixantaine d'entre eux: une trentaine continue dans cette voie. Mais l'activité est saisonnière (automne, printemps, été), il n'y a pas de travail en hiver. Aequitaine puise tout de même dans ce vivier contractuel pour la pleine campagne de labour, lorsque la végétation est en pleine croissance (les adventices et la vigne) et lorsque les traitements se succèdent parfois à huit jours d'intervalle, d'avril à juillet, et aussi d'octobre à novembre. Une bonne dizaine de laboureurs est toujours disponible. Aujourd'hui, trentecinq opèrent dans le Bordelais, dont dix-huit salariés.

Aequitaine a construit un réseau de partenariat avec une vingtaine de châteaux en Bordelais, mais aussi en Bergeracois et dans le Lot-et-Garonne, et cherche à l'élargir. Lors de la dernière formation pour adultes au lycée agricole de Blanquefort, des maitres de stage ont été facilement trouvés dans de nouveaux domaines. L'idée est de les convaincre de créer leur propre équipe, hommes, chevaux et matériel, dans la polyvalence, taille de la vigne en hiver et entretien de la terre avec le cheval le reste de l'année. Les stagiaires trouveraient ainsi leur emploi, à un salaire décent, toute l'année. Mais décrocher un CDI (contrat à durée indéterminée) reste très difficile aujourd'hui ! (fig. 14)

Figure 14

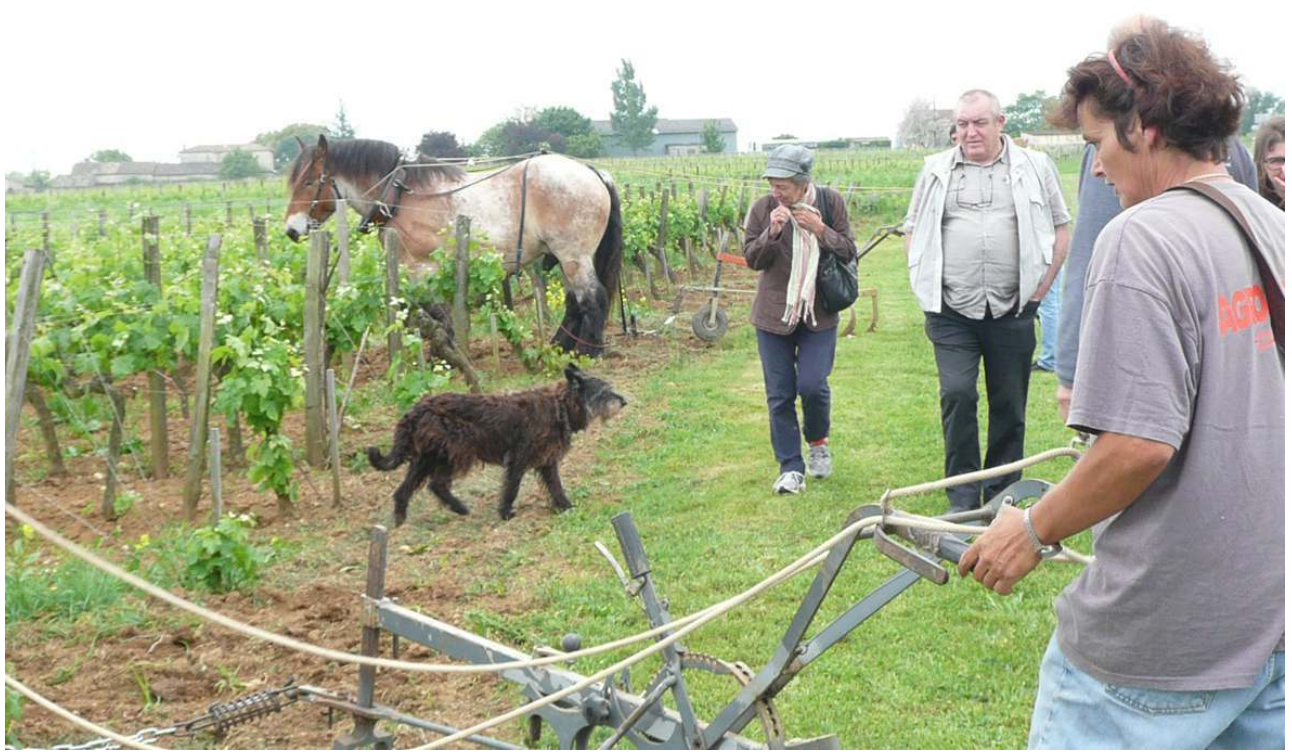

Château Le Puy : discussion entre Anne-Sophie et les participants au colloque de Bordeaux dédié au travail des vignes avec les chevaux, 12 mai 2015.

Phot. Lizet, Bernadette. (c) Bernadette Lizet. 


\section{Conclusion}

Qu'est-ce qui « fait patrimoine » dans ces trajectoires d'hommes et de chevaux de trait ? C'est en premier lieu la diversité des parcours, des combinaisons d'activités, des races animales impliquées, des matériels, des pratiques et des savoir-faire mobilisés, la richesse des relations sociales qui mettent tous ces éléments en cohérence. Il y a du ressort patrimonial également dans la forte motivation à reconstruire un univers de travail que les Trente Glorieuses avaient balayé, dans le désir de s'organiser d'une manière collective pour convaincre autour de soi, transmettre, former, diffuser, démultiplier. La traction animale ressuscitée relève du bien commun sur les registres d'une sauvegarde de la production paysanne, du panel des races, de l'environnement (respecter les sols), de métiers presque disparus, qui donnent sens à l'existence. Mais ressusciter n'est pas conserver à l'identique, ni figer. Bien au contraire : tout est ouvert, interactif et évolutif dans ces aventures familiales ou collectives. Le goût de l'expérimentation et l'esprit de recherche, notamment sur le matériel, sont largement partagés. Dans la transmission familiale, associative ou personnelle, des liens se tissent entre les générations, entre les anciens et les néos, entre les éleveurs et les laboureurs (utilisateurs, débardeurs), entre les sphères de l'attelage de loisir et de l'attelage productif, qui permet d'en vivre. L'une des étonnantes leçons du labourage en Bordelais est l'apparition des laboureuses (35\% des effectifs formés), venues au cheval par l'équitation et qui prennent pied dans un univers d'hommes.

Ce patrimoine du cheval de trait réinventé est riche, mais fragile. La diversité des noms donnés à leur activité par les nouveaux professionnels souligne le caractère économique et culturel de "niches ", le risque d'isolement. La capitalisation des expériences, dans le cadre de formations et de publications en est d'autant plus précieuse. La passion menace souvent de l'emporter sur la solidité du projet économique. L'installation professionnelle reste souvent précaire et l'activité encore saisonnière, comme dans le vignoble bordelais. Un autre risque plane sur la traction animale: le désordre installé dans la filière hippophagique risque d'affaiblir les races, de restreindre le vivier des poulains susceptibles de devenir des travailleurs.

Comment renforcer ces réseaux ? En encourageant les connexions entre les acteurs de la première vague « néo » toujours en mouvement (Hippotese) et ceux de la deuxième vague qui se développe aujourd'hui, notamment dans les vignobles bordelais. D'autres interférences pourraient être provoquées, avec le mouvement mondialisé de l'agroécologie, et aussi celui des vins naturels... L'union fait la force ! C'est dans cet esprit qu'un colloque a été organisé à Bordeaux les 11 et 12 mai 2015, dans le cadre du Salon de l'agriculture Aquitaine, par le "collectif des laboureurs des vignes du Bordelais », la Société française des équidés de travail (SFET) et l'Association des éleveurs de chevaux d'Aquitaine. Intitulé « La vigne, le cheval et l'homme », il était destiné au monde viticole (propriétaires de châteaux notamment) et aux collectivités territoriales. Les témoignages et retours d'expériences très récentes des laboureurs des vignes ont été mis en perspective historique et des liaisons inédites ont été établies entre différents réseaux, du Bordelais à l'Europe. 


\section{NOTES}

1. - Voir LIZET, Bernadette. «Des chevaux de trait pour ménager la nature ». Dans BONNAINDULON, Rolande, CLOAREC, Jacques et DUBOST, Françoise (dir.). Ruralités contemporaines: patrimoine, innovation et développement durable. Paris : L'Harmattan, 2011, p. 190-216.

2. - Voir QUITTET, Edmond et BLANC, Henri. Les races chevalines en France. Paris : la Maison rustique, 1974.

3. - Pour tous ces aspects, voir BÉLIVIER, Julien. La relance du cheval de trait en France (1972-2010), le statut d'un animal en question. Mémoire de master 2 de sociologie, université Paris-Ouest NanterreLa Défense, 2010.

4. - La Route du poisson est une épreuve d'attelage d'endurance, par équipes, réservée aux chevaux de trait. Voir LIZET, Bernadette. Champ de blé, champ de course. Nouveaux usages du cheval de trait en Europe. Paris : Jean-Michel Place, 1997.

5. - Examen fédéral appliqué à l'attelage.

6. - Voir LIZET, Bernadette, BOUJOT, Corinne, BOURDON, Anne et PORTET, François. Chevaux de trait, le retour? Atteleurs, compétition et tradition. Ministère de la Culture, direction du Patrimoine et de l'Architecture, mission du Patrimoine ethnologique. Rapport de fin de recherche. Paris, 1999. Voir sur le site : http://hippotese.free.fr/lizet.sit/titre.htm.

7. - Attelage dit « à la coulalive ».

8. - Ballot de foin d'une centaine de kilos, roulé et serré manuellement dans un filet de corde.

9. - Action de sortir les arbres de la forêt jusqu'au premier chemin carrossable.

10. - On désignait ainsi tous les transports qui se font dans et autour des bâtiments de la ferme (bois, foin, fumier, nourriture des animaux).

11. - Expression classique de marchand, désignant des bêtes de race indéterminée et pas très commodes de caractère. On en trouvait dans toutes les fermes de montagne.

12. - Il s'agissait de groupes de réflexion sur les agricultures " hors normes", principalement localisés dans la région Rhône-Alpes, qui associaient des chercheurs et des paysans. Le premier numéro du bulletin paraît en août 1981. En 1992 (numéro 50), il prend le nom d'Alternatives rurales.

13. - Ingénieur-technicien de formation, Jean Nolle (1918-1993) a contribué à promouvoir la motorisation dans les pays en voie de développement, avant d'en découvrir les effets néfastes. Il change de camp et entreprend de réhabiliter la traction animale, dans une optique moderne et militante, pour assurer l'autonomie des petits paysans. Il a mis au point de nombreux outils innovants et simples à fabriquer.

14. - Voir le résumé qui en a été fait par ROSSIER, E. et JEGO, Y. « Note sur l'utilisation de la traction chevaline en France, premiers résultats d'analyse ». Bulletin du CEREOPA, 1985.

15. - Issu de mouvements para-universitaires de retour à la terre, J.-J. Marquard est passionné de races animales à faibles effectifs, membre fondateur en 1967 du premier Groupement d'étude et de la conservation de la nature en Lorraine (GECNAL). En 1980, il crée l'Association de recherche sur la traction animale et le portage (ARTAP), qui publie un bulletin (une référence technique en traction animale) dont il est le principal rédacteur pendant 10 ans.

16. - « Le chariot bâché », 1987, $n^{\circ} 1$ (réédité en 1994) ; « Harnais agricoles et forestiers », 1988, $\mathrm{n}^{\circ}$ 2 ; « Harnais traditionnels 1886-1908 », 1989, $\mathrm{n}^{\circ} 3$; « Avant-trains modernes à traction animale », 1991, $\mathrm{n}^{\circ}$ 4. Hippobulle: 1986, $\mathrm{n}^{\circ} 1.2014: \mathrm{n}^{\circ} 31$ (une vingtaine de pages en moyenne).

17. - Hippotese et le CERRTA sont des associations régies par la loi 1901. 
18. - Chacun peut les réutiliser librement, en citant simplement la source; mais on ne peut pas les vendre.

19. - Produit du croisement entre l'étalon et l'ânesse.

20. - La double décavaillonneuse Horse one (fabriquée à Margaux par Jean-Yves Lalande, prix de l'innovation à Dethmol en Allemagne) a été mise au point à Latour en 2012, avec les laboureurs employés par Aequitaine et ceux de Château Latour.

21. - Une association de paysans qui a recours aux conseils d'ingénieurs agronomes pour les conforter dans leur schéma biologique.

22. - Une société qui vend du conseil sur la conduite du vignoble en bio.

23. - Organisme de formation en viticulture, notamment pour le «certiphyto" (emploi des produits phytosanitaires).

\section{RÉSUMÉS}

Faire le choix de travailler en France aujourd'hui en s'appuyant sur la force des chevaux implique une motivation patrimoniale à plusieurs titres. Les hommes et les femmes qui ont recours à la traction animale partagent en effet le désir de sauvegarder les races, mais aussi le système constitué par le matériel (le harnachement, les outils attelés) et les compétences mobilisées dans la relation entre les hommes et leurs chevaux. Ces nouveaux professionnels sont animés par le souci de ménager la nature, prendre soin des sols, considérer l'effet de leur action sur les milieux et les espèces. Ils aiment s'immerger dans les paysages qui reflètent cette qualité de lien entre les hommes et le vivant.

Working with horses today, heritage, innovation and transmission. In France today, choosing to rely on horse power involves a heritage motivation at several levels. Men and women who use animal traction share the same desire to save not only the different races of horse but also the system, comprising the equipment used (harnesses, harnessing tools), as well as the skills involved in the relationship between humans and their horses. New professionals are often motivated by their concern to preserve nature, to take good care of the soil and to measure the effects of their work on the environment and the species. They appreciate living in landscapes that reflect the high quality of the relations between humans and other living creatures.

\section{INDEX}

Mots-clés : cheval de trait, traction, laboureur, débardeur, utilisateur, race, matériel, compétence, vivant

Keywords : draft horse, plough horse, stevedores, equipment, living creatures

\section{AUTEURS}

\section{BERNADETTE LIZET}

directrice de recherche honoraire au CNRS bernadette.lizet@orange.fr 
DENY FADY

président d'HIPPOTESE, Paysan-Professeur hippotese@gmail.com

\section{RAMON GARCIA}

laboureur « au cheval », domaine du Prieuré (Saint-Emilion) aequitaine@orange.fr

VINCENT SEÏTÉ

débardeur, utilisateur et meneur de chevaux de trait, EUARL Seïté vincent.seite@bbox.fr 